\title{
L'enseignement de la modalité linguistique en primaire - des voies méthodologiques possibles et souhaitables
}

\author{
José António Costa \\ Polytechnique de Porto - École Supérieure d'Éducation
}

(Text reçu 25 juillet 2019; accepté 25 juillet 2019)

DOI: https://doi.org/10.5565/rev/jt13.810

Résumé : Le fait que les enfants sont exposés dès un âge précoce à des énoncés modalisés (Choi, 2006), exprimant surtout l'obligation et la permission, aurait pu faire croire que ce sujet prenait part à l'école primaire, mais ce n'était pas le cas qu'avant les 12 ans dans plusieurs pays, tels que le Portugal, l'Espagne et la France. Après une caractérisation linguistique de la modalité (van der Auwera \& Plungian, 1998 ; Oliveira \& Mendes, 2013), nous présentons un parcours didactique conçu pour des élèves de $3^{\text {ème }}$ année de l'enseignement primaire, dans lequel on envisage une approche implicite de la modalité dans le but de contribuer à l'éducation linguistique des élèves et à la formation de citoyens informés et socialement compétents (Vieira, 2006). Pour cela, on utilise des méthodologies diversifiées (Arslan, 2010 ; Fernández-Corbacho, 2014 ; Figueiroa-Rego, 2015), de façon à promouvoir des pratiques de réflexion critique sur le monde à travers le langage.

Mots-clés : didactique des langues, modalité linguistique, enseignement implicite, éducation à la citoyenneté, méthodologies communicatives

\begin{abstract}
As children are very early exposed to modalized expressions (Choi, 2006), especially at levels of obligation and permission, it is believed that this content would be more evident in the early stages of Basic Education. However, this does not happen before they are 12 years old in several countries, such as Portugal, Spain and France. Thus, and after having linguistically characterized modality (van der Auwera \& Plungian, 1998; Oliveira \& Mendes, 2013), we present a didactic path conceived to pupils of the $3^{\text {rd }}$ year of primary school, in which we propose an implicit modality approach in order to contribute towards pupil's linguistic education and to the formation of wellinformed and socially competent citizens (Vieira, 2006). Therefore, we make use of different methodological approaches (Arslan, 2010; Fernández-Corbacho, 2014; Figueiroa-Rego, 2015), in order to promote critical reflection on the world through language.
\end{abstract}

Keywords: language didactics, linguistic modality, implicit teaching, citizenship education, communicative methodological approaches

Resumen: El hecho de que los niños están expuestos a los enunciados modalizados desde muy temprano (Choi, 2006), especialmente expresando obligación y permiso, nos hacía creer que este contenido se trabaja ya en las primeras etapas de la Educación Básica. Sin embargo, esto no sucede antes de los 12 años en diversos países, como Portugal, España y Francia. Después de haber caracterizado lingüísticamente la modalidad (van der Auwera \& Plungian, 1998; Oliveira \& Mendes, 2013), presentaremos un curso didáctico diseñado para alumnos del 3er año del 1 er Ciclo de la Educación Básica, con el que proponemos un abordaje implícito de la modalidad con el 
objetivo de contribuir a la educación lingüística de los alumnos y su formación como ciudadanos informados y competentes en la interacción social (Vieira, 2006). Utilizamos así diferentes metodologías (Arslan, 2010; Fernández-Corbacho, 2014; Figueiroa-Rego, 2015) para promover la reflexión crítica sobre el mundo a través el lenguaje.

Palabras clave: didáctica de lenguas, modalidad lingüística, enseñanza implícita; educación a la ciudadanía, metodologías comunicativas

Resum: El fet que els nens estan exposats als enunciats modalitzats des de molt aviat (Choi, 2006), especialment expressant obligació i permís, ens feia creure que aquest contingut es treballa ja en les primeres etapes de l'Educació Bàsica. No obstant, això no succeeix abans dels 12 anys en diversos països, com Portugal, Espanya i França. Després d'haver caracteritzat lingüísticament la modalitat (van der Auwera \& Plungian, 1998; Oliveira \& Mendes, 2013), presentarem un curs didàctic dissenyat per a alumnes del 3r any del 1r Cicle de l'Educació Bàsica, amb el qual proposem un tractament implícit de la modalitat amb l'objectiu de contribuir a l'educació lingüística dels alumnes i la seva formació com a ciutadans informats i competents en la interacció social (Vieira, 2006). Utilitzem així diferents metodologies (Arslan, 2010; Fernández-Corbacho, 2014; Figueiroa-Rego, 2015) per promoure la reflexió crítica sobre el món a través el llenguatge.

Paraules clau: didàctica de llengües, modalitat lingüística, ensenyament implícit, educació per a la ciutadania, metodologies comunicatives

Resumo : Uma vez que as crianças estão, desde muito cedo, expostas a expressões modalizadas (Choi, 2006), especialmente ao nível da obrigação e da permissão, acreditávamos que a modalidade pudesse estar presente no ensino desde os níveis iniciais. No entanto, tal não acontece antes dos 12 anos em vários países, como Portugal, Espanha e França. Assim, e após procedermos a uma caracterização linguística da modalidade (van der Auwera \& Plungian, 1998; Oliveira \& Mendes, 2013), apresentamos um percurso didático concebido para alunos do $3^{\circ}$ ano do $1 .{ }^{\circ}$ Ciclo do Ensino Básico, no qual propomos uma abordagem implícita da modalidade, de forma a contribuir para a formação linguística do aluno e para a formação de cidadãos bem informados e socialmente competentes (Vieira, 2006). Nesse sentido, utilizamos diferentes abordagens metodológicas (Arslan, 2010; Fernández-Corbacho, 2014; Figueiroa-Rego, 2015), a fim de promover uma reflexão crítica sobre o mundo através da linguagem.

Palavras-chave: didática das línguas; modalidade linguística, ensino implícito; educação para a cidadania; metodologias comunicativas.

La modalité est une catégorie grammaticale présente dans plusieurs langues du monde. Elle concerne soit la perspective d'un locuteur face à ce qu'il dit, soit sa croyance en une situation, soit une sorte d'instigation (impositive ou pas) à l'action, soit encore l'identification des 
circonstances qui rendent possible ou nécessaire un certain état de choses (van der Auwera \& Plungian, 1998 ; Oliveira, 2003 ; Nuyts, 2006).

Les enfants sont en contact, dès le plus jeune âge, avec des énoncés modalisés, nommément ceux contenant des ordres, des instructions, des commandes (dimension déontique), des prévisions ou des croyances (dimension épistémique), parmi les actes directifs et expressifs qu'ils reçoivent ou produisent. L'enseignement de la langue maternelle ne profite pourtant pas de ce savoir intuitif, la modalité ne faisant partie des programmes scolaires du Portugal, de l'Espagne et de la France qu'à partir de l'âge de 12 ans, au plus tôt.

Partant de ce constat, on a décidé de mener une étude ${ }^{i}$ que l'on présente dans cet article en trois parties. Tout d'abord, on compare ce qui est prévu au niveau de l'enseignement de la modalité à l'école avec les données issues de la recherche concernant l'acquisition du langage, sachant que l'enfant arrive à saisir l'idée de possibilité et celle de nécessité dès l'âge de cinq ans (Ozturk \& Papafragou, 2014). Deuxièmement, on retrace le scénario linguistique de la modalité, surtout en ce qui concerne les aspects morphologiques, syntaxiques, sémantiques, pragmatiques et discursives à retenir au niveau de l'enseignement. Finalement, on ébauche un plan de cours à poursuivre avec des élèves de la troisième année de l'école primaire où l'on propose l'exploitation d'un album pour enfants. Cette approche pédagogique suit des voies méthodologiques diversifiées et elle permet l'articulation entre l'enseignement de la modalité et l'éducation à la citoyenneté, étant un objectif de l'éducation primaire la promotion de l'esprit critique et de la capacité de se mettre à la place des autres (Santos, 2011), ce que l'on réussira aussi à travers l'étude de la langue dans des situations discursives particulières.

\section{La modalité entre l'acquisition et l'enseignement}

L'idée selon laquelle l'acquisition des langues se situe "à la charnière entre la didactique et la linguistique" (Cuq \& Gruca, 2005, p. 52) permet de soutenir l'investissement que les didacticiens et les enseignants puissent faire dans la connaissance des aspects psycholinguistiques pertinents pour une élaboration plus adéquate de leurs plans de cours. En ce qui concerne la modalité, on tiendra compte de certaines études d'observation naturaliste ou expérimentale ou bien des synthèses produites par des auteurs tels que Stephany (1986), pour l'anglais et pour le grec moderne, et Choi (2006), pour des langues appartenant à différentes familles.

Les modalités non-épistémiques surgissent avant les typologies épistémiques chez des enfants « acquiring typologically and genetically quite different languages » (Stephany, 1986, p. 398). Ces manifestations sont associées à des actes de langage directifs et érotétiques, jouant 
un rôle instrumental pour le bébé qui veut influencer l'adulte, en lui donnant des ordres ou en demandant quelque chose (Stephany, 1983 et 1986). Les premiers actes déontiques apparaissent autour des 18 mois (Stephany, 1983). Tout au long de la troisième année, les enfants arrivent à exprimer la capacité $(2 ; 3)$, l'intention $(2 ; 6)$, la permission, la volonté, l'obligation et la nécessité (Choi, 2006).

Les modalités épistémiques présupposent la distinction entre le réel et la fiction et, par conséquent, « the child's ability to pretend » (Stephany, 1986, p. 393), ainsi bien que leur capacité d'exprimer la futurité, à travers des expressions de doute ou de possibilité. Cela arrive vers la fin de la troisième année (Choi, 2006), étage où l'enfant commence à utiliser l'imparfait modal (Il était une fois ...) si typique des histoires fictionnelles. C'est donc entre les deux et les quatre ans que surgissent les énoncés de prédiction, de certitude et bien d'autres expressions d'incertitude (méconnaissance, possibilité, probabilité et référence hypothétique). Ce n'est qu'autour de la cinquième année que ce type d'énoncés commence à stabiliser dans les productions enfantines, moment où les notions de possibilité et de nécessité sont déjà acquises, ce qui est fondamental pour le développement postérieur des compétences modales des enfants.

La recherche expérimentale remet en question les indications précédentes, en en soulignant « a somewhat later development of epistemic modality » (Choi, 2006, p. 148). En tout cas, pour un enfant de huit ans (l'âge de la plupart des sujets avec qui on a travaillé), la distinction entre différentes forces modales sera déjà acquise, mais l'identification de certains verbes modaux et de différentes valeurs d'un même verbe modal pose toujours des problèmes. Cela va de même pour ce qui concerne la capacité de saisir l'équivalence entre certaines expressions modales ou les divers niveaux d'une gradation modale, surtout épistémique (Tomasello, 2003).

Si les notions modales les plus importantes sont consolidées chez l'enfant vers l'âge de cinq ans, on aurait pu s'attendre à la présence de ce type de sujet dans les programmes scolaires de l'école primaire. L'analyse des documents qui règlementent l'enseignement du Portugais (présentée déjà en Costa, 2016), mais aussi de l'Espagnol et du Français, permet de constater que ce n'est qu'à partir de 12 ans que les élèves commencent à contacter formellement avec cette matière au niveau de la langue maternelle. En fait, on a pu voir, avec surprise, que la modalité peut apparaître plus tôt dans l'enseignement d'une langue seconde (normalement l'anglais, mais aussi dans d'autres cas, selon, par exemple, le Real Decreto 126/2014, qui établit le curriculum de l'éducation primaire en Espagne). Ici, on ne s'occupera que de la L1.

Au Portugal, il y a, en ce moment, deux documents de référence pour l'enseignement de la langue portugaise : Aprendizagens Essenciais (AE) (ME, 2018) et Programas e Metas 
Curriculares de Português (PMC) (Buescu et al., 2015, pour les neuf premières années ; Buescu et al., 2014, pour l'enseignement secondaire, de la dixième jusqu'à la douzième année). On y retrouve des informations différentes.

Les PMC ne placent la modalité qu'à la $12^{\text {ème }}$ année. Le programme pour les trois niveaux de l'enseignement secondaire répartit les plans linguistiques sur plusieurs années, en réservant pour la dixième des thématiques concernant la variation synchronique et diachronique, la syntaxe et la lexicologie ; pour la onzième, des aspects reliés à la linguistique textuelle et à la pragmatique; et, pour la douzième, les questions de sémantique, parmi lesquelles se trouve la modalité et ses types : «modalité épistémique (valeur de probabilité ou de certitude), déontique (valeur de permission ou d'obligation) et appréciative» (Buescu et al., 2014, p. 27). Cette indication attache la modalité uniquement à la sémantique, en oubliant d'autres critères essentiels (comme on le verra par la suite), et surgit très tard par rapport aux informations que l'on a retenues avant. Les $\mathrm{AE}(\mathrm{ME}, 2018)$ font reculer la mention à cette matière vers la 9 ème année, en établissant une liaison avec les données contextuelles : « Utiliser de façon intentionnelle différentes valeurs modales en tenant compte de la situation communicative » (ME, 2018, p. 11). Ce choix règlementaire reprend le scénario que l'on vivait sous les Programmes de 2009 (Reis, 2009), abandonnés en 2014. On y promouvait l'articulation entre les classes de mots, la politesse verbale et les verbes modaux, ce qui permet une vision plus précise et rigoureuse des usages langagiers.

Cette perspective holistique de la modalité au sein de la langue en action est aussi présente dans les programmes en France, où la matière surgit au Cycle 4 (Ministère de l'Éducation nationale, 2018), qui est celui des approfondissements et qui comprend les enfants de 12 à 14 ans. Dans le domaine de l'écriture, poursuivant l'objectif d'adopter des stratégies et des procédures efficaces, on souligne le besoin de «savoir recourir à la modalisation » (Ministère de l'Éducation nationale, 2018, p. 18), à travers l'usage « des indices qui signalent le doute ou la certitude de la part de l'énonciateur (usage modal du conditionnel, verbes modaux : devoir, pouvoir..., adverbes de modalisation, etc.) ». Cette formulation établit un rapport essentiel entre modalité en tant que catégorie grammaticale, mode verbal et classes de mots, en particulier les verbes et les adverbes, ce qui met en relief les façons dont la modalité se projette dans le discours. La section suivante focalise l'importance de cette catégorie au niveau des types de textes, nommément du discours argumentatif, étant la modalisation un procédé possible pour étayer l'argumentation. Dans le domaine consacré à l'étude de la langue, le programme français 
souligne la comparaison d'énoncés pour réfléchir sur les valeurs modales, de façon à saisir les différences issues de situations communicatives particulières.

En Espagne, la modalité ne fait partie que de l'enseignement secondaire. Selon le programme prévu pour le « $1 .^{\circ}$ ciclo de la ESO » (12 ans), dans sa section 3 (Conocimiento de la lengua), il faut exploiter la « reconnaissance, l'usage et l'explication des différents ressources de modalisation en fonction de la personne qui parle ou qui écrit » (Ministerio de Educación, Cultura y Deporte, 2014b, p. 363), ce qui implique, aussi, l'utilisation de temps et modes verbaux particuliers. Il n'y a aucune autre référence à ce sujet dans le domaine de la langue maternelle, étant cette approche un peu vague face à l'ensemble de structures et de situations auxquelles on peut rattacher la modalité.

En somme, les documents portugais les plus actuels privilégient ce rapport étroit entre modalité et mode verbal (à un même niveau que le temps et l'aspect), en reléguant à la marge d'autres critères pertinents, que surtout les programmes français exploitent d'une façon plus systématique. L'exploitation de cette matière plus tôt, avec des enfants de 10 ou 11 ans ou même avant, serait possible et souhaitable, par exemple à partir du moment où l'on commence à analyser le mode subjonctif. On essaye, par la suite, d'élaborer un aperçu des contextes linguistiques où la modalité se fait noter, ce qui va permettre de justifier quelques affirmations que l'on a déjà produites et d'autres qui surgiront après.

\section{La modalité : caractérisation linguistique}

La modalité est une catégorie linguistique qui traduit l'attitude d'un locuteur vis-à-vis d'un énoncé ou d'autres locuteurs, constituant ainsi un phénomène par lequel les structures grammaticales permettent au locuteur de se référer à des situations réelles, hypothétiques ou imaginaires. Cette circonstance situe la modalité, ainsi que la temporalité, dans le champ du déplacement en tant que propriété « that enables natural language to talk about affairs beyond the actual here and now » (von Fintel, 2006, p. 1).

Une définition intensionnelle de cette catégorie concerne les « attitudes différentes du locuteur par rapport à un contenu propositionnel et à son interlocuteur » (Campos, 2004, p. 265). Cette perspective présente l'avantage de combiner la dimension sémantique et la dimension énonciative-pragmatique, dans laquelle la relation entre la proposition et l'énonciateur est également valorisée, c'est-à-dire la relation du dictum, expression du contenu de la pensée, avec le modus, l'attitude du sujet à l'égard de ce contenu (Neves, 2006 ; van der Auwera \& Zamorano Aguilar, 2014).

Selon Campos (2004), une définition extensionnelle de la modalité devient plus complexe, ce qui justifie l'existence de typologies modales très variées (voir synthèse dans Costa, 
2016), normalement réparties en deux grands groupes : celui des modalités épistémiques, concernant la croyance et la connaissance, et celui des modalités non épistémiques, qui touchent à des circonstances qui rendent (im)possible ou (non) nécessaire un tel état de choses, à l'appréciation que l'on fait d'un état de choses ou bien à l'incitation à l'action.

Autrement dit, « epistemics deal with possibilities that follow from the speaker's knowledge, whereas roots deal with possibilities that follow from the circumstances surrounding the main event and its participants » (Hacquard, 2011, p. 1486). Radicales ("roots") est le terme utilisé dans certains contextes pour référer les modalités non épistémiques, qui seront à l'origine, du point de vue historique, des usages épistémiques et qui surgissent en premier dans les productions enfantines. Sous le plan syntaxique, la distinction se fait à travers la place occupée par les modaux dans la structure phrastique : «Epistemic modals are located higher in clausal structure than root modals » (Cinque, 1999, p. 78), ce qui permet de comprendre que ceux-ci puissent se situer sous la portée des modaux épistémiques (mais jamais le contraire).

La caractérisation linguistique de la modalité trouve ses racines dans les apports de la Logique, en particulier des logiciens formels depuis Aristote, systématisés, du côté de la Philosophie, par von Wright (1951) et Rescher (1968) et, de la Linguistique, chez des auteurs tels que Lyons (1977), Angelika Kratzer (1981) ou Palmer (2001). De cela découle la notion de mondes possibles (réels ou imaginaires), sur lesquels les opérateurs modaux agissent et avec lesquels ils établissent une relation d'accessibilité, marquée par deux dimensions : la force (qu'ils expriment une possibilité ou une nécessité) et le type d'interprétation ou saveur modale (Hacquard, 2011), correspondant à la base modale (Kratzer, 1981).

En outre, plusieurs études sur la syntaxe, la sémantique et la pragmatique sont pertinentes pour l'analyse linguistique de la modalité, avec des approches issues de plusieurs orientations théoriques. Ces études conduisent à la conclusion que la modalité peut être considérée comme une supercatégorie (Nuyts, 2005), pour deux raisons. D'un côté, il existe une tendance commune de plusieurs langues à présenter des formes linguistiques exprimant un ensemble de significations modales (les semi-auxiliaires modaux, par exemple), ainsi qu'une relation systématique entre ces significations, à la fois diachroniques et ontologiques. Par ailleurs, toutes les catégories modales peuvent être caractérisées selon l'axe de la possibilité et celui de la nécessité, comme le montre la proposition typologique de van der Auwera et Plungian (1998), que nous présenterons par la suite et qui, pour le portugais, a été adaptée par Oliveira et Mendes (2013) 
Tableau 1. La typologie modale selon van der Auwera et Plungian (1998)

\begin{tabular}{|c|c|c|c|c|}
\hline \multicolumn{3}{|c|}{ Types modaux } & Possibilité & Nécessité \\
\hline \multicolumn{3}{|l|}{ épistémique } & possible & probable \\
\hline \multirow{3}{*}{$\begin{array}{l}\text { non } \\
\text { épistémique }\end{array}$} & \multicolumn{2}{|c|}{ interne au participant } & capacité & nécessité interne \\
\hline & \multirow{2}{*}{$\begin{array}{l}\text { externe } \\
\text { au participant }\end{array}$} & déontique & permis/défendu & obligatoire \\
\hline & & non déontique & conditions possibles & conditions nécessaires \\
\hline
\end{tabular}

On remarque, tout d'abord, la distinction entre modalités épistémiques et non épistémiques (Tableau 1) que l'on avait référée comme étant centrale dans une typologie modale. Le type épistémique concerne la croyance en un état de choses (ex : Je dois aller au cinéma). La modalité interne au participant implique la capacité (ex : Il sait parler six langues.) ou la nécessité interne (ex : Je dois aller chez le médecin.). La modalité externe au participant recouvre les conditions externes qui rendent possible ou nécessaire une situation et qui peuvent être déontiques (ex : Tu dois respecter la loi. / Il faut absolument que tu respectes la loi.), d'une façon plus ou moins impositive, mais concernant toujours l'incitation à l'action, ou non déontiques (ex : Il faut prendre le métro pour arriver au centre-ville. / Tu peux arriver au Tribunal à pied ou en métro).

Bien qu'il y ait plusieurs typologies possibles, on a décidé de suivre la proposition de van der Auwera et Plungian (1998) parce que, outre la possible organisation en deux types déjà signalée, on arrive aussi à saisir une relation particulière d'hyponymie / hyperonymie entre les modalités déontique et externe au participant, permettant l'évaluation des affinités et des différences entre l'obligation et les circonstances externes. En plus, cette typologie permet de retracer des liens historiques (modalité externe au participant déontique > modalité externe au participant non déontique, par exemple) et/ou métaphoriques ou métonymiques (obligation déontique > obligation non déontique présente, par métaphore, dans un exemple tel que Il faut qu'il fasse beau. Je n'ai pas de parapluie.).

Ces liens sont représentés dans des cartes sémantiques que van der Auwera et Plungian (1998) ont esquissées, de façon à rendre compte non seulement des types modaux, mais aussi des rapports qu'ils établissent et de l'évolution qu'ils ont connue, parfois à partir d'autres valeurs non modales, telles que la futurité ou l'intention. On y aperçoit donc un parcours de (non) grammaticalisation ${ }^{\mathrm{ii}}$, phénomène sous-jacent aux usages modaux si l'on envisage la modalité comme la «grammaticalisation des attitudes et des opinions des locuteurs » (Oliveira, 2003, p. 243). Cette grammaticalisation se produit progressivement (cline) et peut impliquer des 
processus d'affaiblissement sémantique, une dépendance syntaxique accrue et une sorte de friction morphophonologique (Lehmann, 2002). Parfois, celle se vérifie en diachronie ; parfois, les usages à différentes valeurs coexistent dans un même état de langue. Elle rend compte en tout cas d'un processus de subjectification et de intersubjectification (Portner, 2009 ; Narrog, 2012), par lequel le locuteur prend une place plus importante dans la mesure où il fait prévaloir son point de vue ou sa volonté (ex : Tu dois aller chez le médecin.) ou bien il implique son interlocuteur dans un état de choses (ex : Peux-tu me passer le sel ?). La subjectification permet d'expliquer l'utilisation épistémique d'une structure déontique, tandis que l'intersubjectification se trouve à l'origine des usages de politesse.

La modalité constitue donc une catégorie grammaticale qui établit un rapport étroit avec d'autres, telles que la temporalité, ce qui aide à comprendre que la modalité soit présente dans plusieurs structures et usages linguistiques :

- dans des actes discursifs expressifs (opinions, croyances) et dans des actes injonctifs (ordre, demande, suggestion, permission, interdiction);

- dans différentes classes de mots (noms, verbes, adjectifs), affixes, temps et modes verbaux ;

- dans des structures syntaxiques particulières (par exemple, des structures telles que $I l$ est possible que / Il se peut que ...);

- dans des expressions d'atténuation et d'intensification (ex : Je vais peut-être au congrès. | Je vais au congrès. | Je vais au congrès, bien sûr !) ;

- dans le principe conversationnel de la politesse (ex : Pourriez-vous me préparer un café?).

Parfois, ce n'est qu'à travers l'intonation que l'on arrive à identifier certaines nuances modales dans les énoncés, ce qui ouvre un autre domaine d'étude de la modalité vers le champ de la prosodie et des types et formes de phrases, étant donné, par exemple, la valeur déontique de certains énoncés impératifs ou le degré de certitude des déclarations. D’ailleurs, la négation et la condition, en tant qu'opérateurs de restriction, jouent aussi un rôle important au sein de la modalité. La première déclenche des relations d'équivalence particulières (Horn, 1978 ; Oliveira \& Mendes, 2013) : ne pas pouvoir (faire qqc) = devoir ne pas (faire qqc); ne pas devoir (faire qqc) = pouvoir ne pas (faire qqc). La deuxième peut réduire la force modale d'un énoncé, comme on le constate dans : Tu dois venir. / Tu dois venir s'il pleut. / Tu dois venir si tu veux.

La modalité se trouve, en effet, au centre de plusieurs usages langagiers. On a essayé d'identifier ses caractéristiques les plus frappantes et les situations communicatives où elle se 
fait noter. Cet ancrage discursif de la modalité dans des structures linguistiques diversifiées et dans certains actes et genres discursifs (ainsi bien que sa présence dans le quotidien des enfants) justifie une approche didactique dès les premières années de la scolarité, comme on essayera de le montrer dans la section qui suit.

\section{La modalité dans l'enseignement primaire : parcours didactique et ap- proches méthodologiques}

Cette approche pédagogique de la modalité comprend une séquence didactique pour mener les élèves ( $3^{\text {ème }}$ année de l'école primaire au Portugal ; âge : huit ans) à discuter de la thématique du vol, en lisant la version portugaise de l'album This is not my hat, de l'illustrateur canadien Jon Klassen (2015).

Outre la promotion de la lecture de textes littéraires, on a envisagé l'occasion de mettre les enfants en contact avec un ouvrage dont l'exploitation présente plusieurs avantages. Tout d'abord, et pour ce qui est de notre sujet, on y trouve des expressions déontiques et épistémiques, mais aussi des situations communicatives qui peuvent déclencher leur utilisation par les élèves. Une telle utilisation surgirait spontanément lors des débats ou serait évoquée dans la réponse écrite aux questions de reconnaissance, d'inférence (qui se prêtent à un plus grand degré d'objectivation et favorisent la reconnaissance ou l'utilisation d'expressions modales) ou d'appréciation (qui permettent aux étudiants d'utiliser spontanément certaines structures modales afin de pouvoir diversifier le type de productions, ce qui nous mène à ébaucher une image plus complète de la compétence linguistique des élèves au niveau de la modalité). Cela dit, on aurait pu créer des conditions pour que les enfants aillent au-delà des jugements de valeur sans fondement, évitant la seule production de mots comme bon, mauvais, bien, mal, vides de sens quand ils sont décontextualisés et remplacent un raisonnement et une réflexion plus profonde sur diverses circonstances de la vie.

Une autre dimension pertinente concerne l'illustration et le type de dialogue (Baptista, 2009) établi avec le texte verbal (conflit, complémentarité, éloignement, paradoxe). Chez Jon Klassen, l'utilisation du paradoxe entre ce que l'image et le texte véhiculent est fréquente, ce qui permet, par l'humour (parfois sombre, voire noire), d'attirer l'attention des élèves sur des aspects particuliers du récit, tels que l'état d'esprit des personnages ou leurs réactions face à certaines péripéties. Plusieurs images fonctionnent dans ce contexte comme des indices dirigeant l'attention, la lecture et l'interprétation des séquences : l'inclinaison de la végétation, le regard des animaux, le crabe qui pointe et les bulles d'eau suggèrent la direction prise par le 
poisson qui vient de passer et favorisent la reconstitution des étapes du récit et des décisions prises par les personnages.

Le travail que l'on a mené avec des élèves de primaire envisage aussi l'articulation entre la compréhension textuelle et les représentations des enfants sur le monde, ce qui peut aider à promouvoir l'éducation à la citoyenneté et la construction de l'identité des sujets. En effet, les enfants doivent produire des opinions soutenues, évaluer des hypothèses sur l'évolution de l'histoire ou des scénarios concernant la vie courante (Carvalho, 2013). En ce sens, les enseignants et les élèves sont considérés comme des consommateurs critiques et des producteurs créatifs de connaissances dans le contexte de la négociation pédagogique (Jiménez Raya et al., 2007).

On présente ensuite les étapes didactiques essentielles et les méthodologies sous-jacentes à cette séquence didactique. Il y a une phase de pré-lecture destinée à mettre les enfants en contact avec l'ouvrage et une phase de lecture divisée en six moments. Les étapes didactiques seront résumées dans des tableaux, précédés d'un bref texte d'introduction.

À une époque où les enfants sont entourés d'images en mouvement et développent des stratégies spécifiques pour la perception et le traitement de ces images, il devient encore plus important, en ce qui concerne les images fixes, de « promouvoir des activités de lecture systématique attirant l'attention des enfants pour la nécessité de lire certains détails qui peuvent passer inaperçus et de mettre en relation certains éléments qui gagnent en sens quand on se rend compte de la raison de la relation qui les a rapprochés » (Baptista, 2014, p.63). Cette idée constitue le point de départ de la phase de la pré-lecture.

Tableau 2. Les activités de pré-lecture : parcours

Les élèves ferment les yeux et posent la tête sur la table. L'enseignant énonce des indices sur ce qu'ils
vont entendre (il parle dans un espace où il y a de l'eau, de la végétation, du sable, des poissons ...) et
met en arrière-plan le son de la mer. Les élèves ouvrent les yeux et remarquent la couverture de l'ouvrage
projetée sur le tableau.
Q: Nous voyons un poisson avec un chapeau. Le titre est Este chapéu não é meu. Dans quelles situations
utilisons-nous quelque chose qui ne nous appartient pas ? | A: On a peut-être volé / emprunté ... (ré-
ponses faites oralement). [Q - question; A - réponses possibles]
L'enseignant projette les pages 7 et 8 et attire l'attention sur le gros poisson. Il lit ensuite la phrase dite
par le petit poisson, en demandant : Est-ce que l'on sait maintenant comment le petit poisson a obtenu
le chapeau? En ce moment, l'enseignant distribue la fiche $\mathrm{n}^{\circ} 1$, où il y a trois hypothèses de réponse.
Les élèves marquent d'une croix la réponse qu'ils jugent correcte. L'enseignant recueille les fiches et
demande aux élèves de justifier oralement leurs réponses, menant le dialogue à l'intensité modale de
verbes tels que pouvoir ou devoir (en portugais : poder, dever e ter de).
On regarde donc la quatrième de couverture et confirme le vol du chapeau, ce qui motive un débat sur
le vol (les possibles raisons, la validité et le poids des raisons).

Nous avons décidé d'organiser la phase de lecture en six moments, correspondant aux six plans textuels que nous avons individualisés en fonction de la progression du récit, mais également aux expectatives que nous avons créées vis-à-vis de l'utilisation d'énoncés modaux 
par les élèves dans leur analyse. On espérait donc que ils soient capables de percevoir les schémas constitutifs du texte, étant un rôle de l'école celui de promouvoir chez l'enfant « la familiarité avec des plans fixes, pertinents à la fois d'un point de vue symbolique et culturel (...) et du point de vue de l'intégration sociale et professionnelle (...); mais c'est aussi à elle de stimuler la capacité de reconnaître / d'établir des plans occasionnels »(Coutinho, 2011, p. 200). Dans le tableau contenant les moments de la lecture, on insère aussi les compétences à poursuivre à chaque étape.

Tableau 3. Les activités de lectura : parcours et compétences

\begin{tabular}{l} 
Parcours \\
\hline $\boldsymbol{Q}$-question/affirmation de l'enseignant; $\boldsymbol{A}$-réponses possibles des élèves \\
Moment 1 \\
Le professeur lit les pages 1 à 4. \\
"Et peut-être qu'il ne se réveille pas si tôt » (p.6) (lire sans projeter l'image). \\
L'enseignant demande aux élèves d'indiquer une autre façon de dire cette \\
phrase. Il fournit trois hypothèses dans une fiche ( $\mathrm{n}^{\circ}$ 2) et les élèves signalent \\
ce qu'ils considèrent correct. \\
Les pages 6 à 10 sont lues par l'enseignant, projetant les illustrations sans texte \\
associé. Soit par la réaction spontanée des élèves, soit par des questions po- \\
sées, l'enseignant dirige la lecture afin d'explorer le paradoxe entre le texte \\
verbal et l'image. \\
L'enseignant analyse les illustrations avec les élèves pour tirer des conclu- \\
sions sur l'état d'esprit des personnages et sur les expressions linguistiques et \\
les marques non linguistiques qui le transmettent - Compréhension inféren- \\
tielle.
\end{tabular}

\section{Moment 2}

Devant la phrase présente aux pages 11-12, on pose la question (d'appréciation): Q: Le gros poisson aura-t-il un moyen de savoir qui a pris le chapeau? A : Non. / Oui, s'il arrive à trouver le petit poisson / Oui, si quelqu'un qui l'ait vu le lui raconte.

L'enseignant lit les pages 13-15.

\section{Moment 3}

L'enseignant projette l'illustration de la page 16 et pose des questions (de reconnaissance, d'inférence et d'appréciation) :

Q: Qui a vu passer le poisson rouge? Comment savons-nous qu'il est parti ? L'enseignant distribue la fiche $\mathrm{n}^{\circ} 3$ avec trois scénarios (pode, deve, tem de) qui expriment trois hypothèses de comportement. Les élèves les organisent dans une séquence graduée.

L'enseignant encourage le débat en posant des questions telles que :

Q: Le crabe peut-il être sûr que le petit poisson a volé le chapeau? Devrionsnous dénoncer quelqu'un sur la base de l'apparent si nous ne sommes pas sûrs qu'il ait commis un vol ? Raconteriez-vous au gros poisson où le petit poisson se trouve? Devrions-nous toujours garder un secret, même sous promesse de rien raconter?

\section{Moment 4}

$\mathbf{Q}$ : Voyons ce que le crabe a décidé de faire.

L'enseignant projette les pages 17 et 18 , avec juste l'illustration, et lit la phrase prononcée par le narrateur ( « Je ne suis donc pas inquiet»).

Puis il demande : $\mathbf{Q}:$ Le petit poisson a-t-il des raisons de s'inquiéter?

\section{Moment 1 \\ L'élève produit des in- férences sur le texte à partir des images et de leur rapport avec le texte verbal, en utilisant des structures épisté- miques.}

\section{Moment 2}

L'élève répond à des questions de compréhension à travers des expressions modales, exprimant parfois des conditions.

\section{Moment 3}

L'élève produit des énoncés servant à évaluer le comportement des personnages ou des situations du quotidien, à travers des expressions déontiques et épistémiques.

\section{Moment 4}

L'élève produit des inférences à partir des images pour arriver à comprendre le texte. 
Q : Quels éléments de l'illustration justifient votre réponse? (des questions de soutien sont disponibles si les élèves ont du mal à bien répondre ; par exemple : Vers où pointe le crabe? À quel autre moment avons-nous vu le gros poisson avec le regard tel qu'il apparaît ici? Que ressentait-il alors?) Q: Pourquoi le crabe décide-t-il de dire la vérité? A: De peur du gros poisson. / Parce qu'il croit qu'il ne faut pas mentir. / Parce qu'il n'est pas un ami du petit poisson. / Parce qu'il ne se soucie pas de ce qui va se passer.

\section{Moment 5}

Le professeur lit la page 20 .

$\mathbf{Q}$ : Comment vit-on avec une conscience coupable?

L'enseignant distribue la fiche $n^{\circ} 4$, avec des phrases exprimant des valeurs déontiques et épistémiques, que les élèves doivent identifier, en saisissant aussi l'ambiguïté présente dans un énoncé.

\section{Moment 6}

Le professeur lit les pages 21-26, en projetant des images sans projeter le texte.

Enfin, l'enseignant présente les trois dernières images qui apparaissent dans l'œuvre et qui ne sont pas accompagnées de texte.

Illustration $1-\mathbf{Q}$ : Et maintenant que les deux traversent la végétation, le gros poisson trouvera-t-il le petit? Lorsque les autres ne savent pas que nous avons commis une erreur, cette erreur existe-t-elle toujours? Et s'ils ne savent pas où nous trouver, sommes-nous toujours coupables? Ou seuls ceux qui sont pris sont vraiment coupables? Voyons donc si le petit poisson a été trouvé.

Illustration 2 - Q : Nous voyons le gros poisson qui revient. Aura-t-il récupéré son chapeau?

Illustration 3-Q : Maintenant on sait qu'il l'a récupéré. Que sera-t-il arrivé au petit poisson?
L'élève commente les actions des personnages, en utilisant des structures déontiques et épistémiques.

\section{Moment 5}

L'élève évalue le quotidien à travers les situations du récit.

L'élève identifie des expressions modales et leurs valeurs.

\section{Moment 6}

L'élève évalue le quotidien à travers les situations du récit.

L'élève essaye d'anticiper la fin de l'histoire, à travers des expressions épistémiques.

L'approche que l'on vient de proposer tend à favoriser une participation active au débat des élèves, dans le respect des idées des autres et avec le soutien de leurs propres opinions, en valorisant à plusieurs reprises les structures linguistiques typiques du discours déontique, lors de l'évaluation critique des actions, mais également celles de nature épistémique, lorsque nous considérons les conséquences positives et négatives de nos actions. Bien que moins fréquentes, il existe également des expressions qui transmettent les modalités interne ou externe au participant.

Au niveau des méthodologies, les pédagogies critiques et l'hyper-pédagogie critique (Cruz, Araújo e Sá \& Moreira, 2011) servent les buts énoncés ci-dessus, en promouvant dès le plus jeune âge des pratiques de réflexion critique et de résolution de problèmes, des procédures rendues encore plus visibles par la philosophie pour enfants (Figueiroa-Rego, 2015). Cette méthodologie fondée par Lipman (1990) valorise la capacité à poser des questions sur le monde et à évaluer les conséquences de nos actions, plutôt qu'à trouver des réponses fermées et souvent acritiques. Une telle démarche permet encore de contribuer à l'autonomie des élèves, étant la 
pédagogie critique aussi une pédagogie à l'autonomie, dans la mesure où elle implique une " pratique éducative qui se situe entre le réel et l'idéal, en instituant des espaces du possible » (Vieira, 2006, p. i).

Certaines étapes didactiques de ce parcours s'inspirent également du multisensory learning / l'approche multisensoriel (Arslan, 2010), en favorisant le contact de l'étudiant avec différents stimuli sensoriels (auditifs et visuels), parfois en conflit au sein de l'intrigue textuelle. La confrontation entre différents inputs stimule le développement cognitif et pose des défis aux élèves, qui doivent les résoudre pour avancer dans l'histoire, ce qui peut prendre, dans l'approche didactique, une forme gamifiée (Foncubierta \& Rodríguez, 2015), technique consistant dans la mobilisation de la dynamique et des éléments propres du jeu (limites de temps, ponctuation, dés) pour l'enseignement des langues, sans pour autant transformer les classes en jeux.

Nous suivons également certaines prémisses de l'approche communicative expérientielle, qui « supone la conexión de los contenidos de lengua con el estudiante, sus aficiones e intereses y con su experiencia del mundo ; ayudándole, poco a poco, a autodirigir su propio aprendizaje »(Fernández-Corbacho, 2014, p. 1), en particulier aux moments où nous avons demandé aux élèves d'évaluer le comportement des personnages et leur relation avec des situations de la vie quotidienne.

Enfin, dans l'esprit d'une didactique du compromis (Bronckart, 2007), nous nous sommes concentrés sur la combinaison de procédures typiques du laboratoire de grammaire (Duarte, 1992), qui met l'accent sur la découverte guidée de régularités linguistiques, avec certains principes de la pédagogie des discours (Fonseca, 1988-89), qui préconise l'évaluation de ces régularités dans la projection des textes et des situations d'usage réelles, en tenant compte des coordonnées énonciatives-pragmatiques inhérentes. Il s'agit d'une perspective d'association de stratégies déjà testée, par exemple, dans l'enseignement du portugais (Silvano \& Rodrigues, 2010) et de l'espagnol (Camps, 2009).

En préconisant la combinaison de différentes méthodologies dans l'enseignement des langues, nous espérons promouvoir l'autonomie future de l'élève en lui fournissant, sous une perspective constructiviste et cognitiviste, « tous les moyens possibles pour qu'il puisse accompagner et monitorer les pas et les progrès de ses connaissances linguistiques, en même temps qu'il les acquiert » (Pinto, 2009, p. 88). 


\section{Quelques remarques finales}

La modalité est une catégorie linguistique complexe, mais la présence des structures modales dès très tôt dans le quotidien des enfants et le rapport étroit avec d'autres catégories et usages linguistiques légitiment qu'elle soit enseignée déjà en primaire, même si les programmes scolaires ne le prévoient qu'après les 12 ans.

Le parcours didactique que l'on vient de présenter a été mis en place dans quatre groupes d'élèves de l'enseignement primaire (Costa, 2016). On a pu constater que ces enfants ont été plus forts que ceux du groupe de contrôle au niveau de l'identification de la valeur de probabilité du verbe devoir, de la lecture déontique de l'imparfait et de l'identification de l'expression modale la plus intense. Ils arrivent aussi à modaliser leur discours en utilisant des actes discursifs non impositifs et des structures syntaxiques plus complexes. La prévalence d'un discours plutôt épistémique et modalisé au détriment des jugements de valeur catégoriques en est aussi une marque importante.

Cela dit, on croit possible ce type d'approche didactique, pourvu qu'il soit articulé avec l'étude d'autres matières typiques de ces années (les classes de mots, la politesse, le temps et le mode verbal...), dans un esprit de promotion de l'éducation à la citoyenneté. On espère donc former des citoyens critiques et actifs qui fuient les attitudes manichéennes de division du monde en bien et en mal, en exprimant leur propre point de vue, mais en acceptant également les différentes visions du monde sur un sujet quelconque.

\section{Références bibliographiques}

Arslan, K. (2010). Multisensory learning and the future of teaching. Ezine Articles. Disponível em http://ezinearticles.com/?Multisensory-Learning-and-the-Future-ofTeaching\&id=4077370. Acesso em 23 dez. 2015.

Baptista, M. A. (2009). Interacções texto/imagem: o caso particular da legenda de fotografia. Lisboa: FCG / FCT.

Baptista, M. A. (2014). Literacia visual no jardim de infância. As imagens ao pé das letras. In F. L. Viana et al. (coord.). Ler para ser. Os caminhos antes, durante e... depois de aprender a ler. Coimbra: Almedina.

Bronckart, J-P. (2007). La enseñanza de lenguas: para una construcción de las capacidades textuales. In J-P. Bronckart (2007). Desarrollo del lenguaje y didáctica de las lenguas. Madrid e Buenos Aires: Minõ y Dávila editores. 
Buescu, H., Maia, L. C., Silva, M. G. \& Rocha, M. R. (2014). Programa e Metas Curriculares de Português do Ensino Secundário. Lisboa: MEC e DGE.

Buescu, H., Morais, J., Rocha, M. R. \& Magalhães, V. F. (2015). Programa e Metas Curriculares de Português do Ensino Básico. Lisboa: MEC e DGE.

Campos, M. H. C. (2004). A modalidade apreciativa: uma questão teórica. In F. Oliveira \& I. M. Duarte (org.). Lingua e Discurso - Homenagem a Joaquim Fonseca. Universidade do Porto, Novembro de 2003.

Camps, A. (2009). Actividad metalinguística y aprendizaje de la gramática: hacia un modelo de enseñanza baseado en la actividad reflexiva. Cultura y Educación, 21 (2), 199-213.

Carvalho, J. A. B. (2013). A escrita na escola: uma visão integradora. Revista Interacções, 9 (27), 186-206. ISSN: 1646-2335.

Cinque, G. (1999). Adverbs and functional heads. A cross-linguistic perspective. Oxford: Oxford University Press.

Choi, S. (2006). Acquisition of modality. In W. Frawley (ed). The expression of modality. Berlin: Mouton de Gruyter.

Costa, J. A. (2016). As pontes entre Didática e Linguística: o possível e o necessário. Contributos da Linguística sincrónica e diacrónica para o ensino implícito da modalidade e dos verbos modais no $1 .{ }^{\circ}$ CEB. Porto: FLUP [Tese de Doutoramento não publicada]

Coutinho, M. A. (2011). Macroestruturas e microestruturas textuais. In I. Duarte \& O. Figueiredo (org.). Português, Lingua e Ensino. Porto: Universidade do Porto.

Cruz, M.; Araújo e Sá, M. H. \& Moreira, A. A. (2011). Duma pedagogia crítica a uma hiperpedagogia crítica: caminhos para a autonomia no processo de ensinoaprendizagem on-line. In J. L. C. Silva et al. (orgs.). Actas do Congresso Ibérico Pedagogia para a Autonomia. 5. ${ }^{\circ}$ Encontro do GT-PA (pp. 137-150). Braga: Universidade do Minho. ISBN: 978-989-8525-02-4.

Cuq, J-P. \& Gruca, I. (2005). Cours de didactique du français langue étrangère et seconde. Grenoble: Presses Universitaires de Grenoble.

Duarte, I. (1992). Oficina gramatical: contextos de uso obrigatório do Conjuntivo. In M. R. Delgado-Martins et al. (org.). Para a Didáctica do Português. Seis Estudos de Linguística (pp. 165-177). Lisboa: Edições Colibri.

Fernández-Corbacho, A. (2014). Aprender una segunda lengua desde un enfoque comunicativo experiencial. Programa de Desarrollo Profesional. Madrid: Editorial Edinumen. 
Figueiroa-Rego, M. J. (2015). Filosofia com Crianças e Jovens. Livro do Professor (Nível 1, 5/7 anos). Lisboa: Edições Piaget.

Foncubierta, J. \& Rodríguez, C. (2015). Didáctica de la gamificación en la clase de español. Programa de Desarrollo Profesional. Madrid: Editorial Edinumen.

Fonseca, J. (1988-89). Ensino da língua materna como pedagogia dos discursos. Diacrítica, 34, 63-77.

Hacquard, V. (2011). Modality. In C. Maienborn et al. (ed.). Semantics: An International Handbook of natural language meaning. Berlin: Mouton de Gruyter.

Horn, L. R. (1978). Remarks on neg-raising. In P. Cole (ed.) Syntax and Semantics. (Vol. 9, pp. 129-220). New York: Academic Press.

Jiménez Raya, M., Lamb, T. \& Vieira, F. (2007). Pedagogy for Autonomy in Language Education in Europe - Towards a framework for learner and teacher development. Dublin: Authentik.

Klassen, J. (2015). Este chapéu não é meu (tradução portuguesa). Lisboa: Orfeu Negro (Original publicado em 2012).

Kratzer, A. (1981). The notional category of modality. In H. J. Eikmeyer \& H. Rieser (eds.). Words, worlds, and contexts: New approaches in word semantics (pp. 38-74). Berlin: Mouton de Gruyter.

Lehmann, C. (2002). Thoughts on grammaticalization (2nd ed.). Edição do autor.

Lipman, M. (1990). A filosofia vai à escola (trad. portuguesa, 3. a ed.). São Paulo: Summus.

Lyons, J. (1977). Semantics (vol. 2). Cambridge: Cambridge University Press.

Ministère de l'Éducation nationale (2018). Programmes d'enseignement, Cycle 4, modification, arrêté du 17-7-2018, Annexe 3. Le Bulletin officiel de l'Éducation nationale, 30, du 26-7-2018.

Ministério da Educação (2018). Aprendizagens Essenciais de Português (9. ${ }^{\circ}$ ano). Lisboa: Ministério da Educação.

Ministerio de Educación, Cultura y Deporte (2014a). Real Decreto 126/2014, de 28 de febrero, por el que se establece el currículo básico de la Educación Primaria. Boletín Oficial del Estado, 52, de 1 de marzo de 2014. Referencia: BOE-A-2014-2222.

Ministerio de Educación, Cultura y Deporte (2014b). Real Decreto 1105/2014, de 26 de diciembre, por el que se establece el currículo básico de la Educación Secundaria Obligatoria y del Bachillerato. Boletín Oficial del Estado, 3, de 3 de enero de 2015. Referencia: BOE-A-2015-37. 
Narrog, H. (2012). Modality, subjectivity and semantic change. A cross-linguistic perspective. Oxford: Oxford University Press.

Neves, M. H. (2006). A modalidade. In: I. V. Koch (org.). Gramática do português falado (2. ed., vol. 6, pp. 171-208). São Paulo: Unicamp / FAPESP.

Nuyts, J. (2006). Modality: overview and linguistic issues. In W. Frawley (ed). The expression of modality. Berlin: Mouton de Gruyter.

Oliveira, F. (2003). Modo e Modalidade. In M. H. M. Mateus et al. (org.). Gramática da Língua Portuguesa (pp. 243-272). Lisboa: Editorial Caminho.

Oliveira, F. \& Mendes, A. (2013). Modalidade. In E. B. P. Raposo et al. (orgs.). Gramática do Português (Vol. 1, pp. 623-669). Lisboa: Fundação Calouste Gulbenkian

Ozturk, O. \& Papafragou, A. (2014). The acquisition of epistemic modality: from semantic meaning to pragmatic interpretation. Language Learning and Development, 00, 1-24. DOI: $10.1080 / 15475441.2014 .905169$.

Palmer, F. R. (2001). Mood and Modality (2nd edn). Cambridge University Press.

Pinto, M. G. (2009). Ensino da língua (portuguesa): uma profissão com futuro. In I. M. Duarte et al. (org.). A linguagem ao vivo. Porto: FLUP (Original publicado em 2004).

Portner, P. (2009). Modality. Oxford: Oxford University Press.

Reis, C. (coord.) (2009). Programas de Português do Ensino Básico. Lisboa: ME e DGIDC.

Reschner, N. (1968). Topics in philosophical logic. Dordrecht: Reidel.

Santos, M. E. B. (2011). Educação para a cidadania. Proposta curricular para os Ensinos Básico e Secundário. (documento de trabalho).

Silva, A. S. (2015). Subjetificação, objetificação e (des)gramaticalização nas construções completivas infinitivas em português, em comparação com outras línguas românicas. In: G. Engwall \& L. Fant (eds.) Festival Romanistica. Contribuciones lingüisticas Contributions linguistiques - Contributi linguistici - Contribuições linguísticas (pp. 64-91). Stockholm Studies in Romance Languages. Stockholm: Stockholm University Press. DOI: http://dx.doi.org/10.16993/bac.d. License: CC-BY.

Silvano, P. \& Rodrigues, S. (2010). A Pedagogia dos Discursos e o Laboratório Gramatical no ensino da gramática. Uma proposta de articulação. In A. M. Brito (org.). Gramática: História, Teorias, Aplicações (pp. 275-286). Porto: FLUP.

Stephany, U. (1983). The development of modality in language acquisition. Arbeitspapier, $\mathrm{n}{ }^{\circ}$ 43. Institut für Sprachwissenschaft, Universität Köln. 
Stephany, U. (1986). Modality. In P. Fletcher \& M. Garman (eds). Language acquisition: studies in first language development. Cambridge: Cambridge University Press

Tomasello, M. (2003). Constructing a language. A usage-based theory of language acquisition. Cambridge, Massachussets \& London: Harvard University Press.

van der Auwera, J. \& Plungian, V. (1998). Modality’s semantic map. Linguistic Typology, 2, $79-124$

van der Auwera, J. \& Zamorano Aguilar, A. (2014). The history of mood and modality. In J. Nuyts \& J. van der Auwera (eds). The Oxford Handbook of Mood and Modality. Oxford: Oxford University Press.

Vieira, F. (2006). Pedagogia para a autonomia: construir uma prática re(ide)ealista. In F. Vieira (org.). Cadernos 4. GT-PA. Braga: Universidade do Minho.

von Fintel, K. (2006). Modality and Language. In D. M., Borchert (org.). Encyclopedia of Philosophy (2nd edn). MacMillan.

von Wright, G. H. (1951). An Essay in Modal Logic. Amsterdam: North-Holland Publishing Company.

\footnotetext{
${ }^{i}$ Cette étude a été menée pendant l'élaboration de notre thèse de Doctorat en Didactique des Langues (Costa, 2016). Ici, on présente surtout les questions concernant les voies méthodologiques exploitées et moins les résultats du travail avec les élèves de quatre écoles primaires de Porto, dont on ne rendra compte que quand il se révèle pertinent.

ii Pour une discussion sur les parcours de grammaticalisation et de dégrammaticalisation autour des usages modaux, voir Silva (2015).
}

\begin{abstract}
Author's information
José António Costa is Associate Professor at the School of Education within the Polytechnic Institute of Porto, teaching, since February 2000, several subjects concerning the areas of Descriptive Linguistics, Language Acquisition, Historical Linguistics, Applied Linguistics and Language Didactics. He holds a PhD in Language Sciences, specialization area of Language Didactics (2016), by the Faculty of Arts of the University of Porto, an institution where he previously completed his Master's Degree in Linguistics (2010) and the Degree in Modern Languages and Literatures, Portuguese and French Studies (1995). He develops research in the areas of Historical Linguistics, Applied Linguistics to Language Teaching and Language Didactics, in subjects such as orthography, grammatical gender, lexical relations and modality, in a linguistic (synchronic and diachronic) and didactic perspective.

E-mail: joseacosta@ese.ipp.pt

Pour citer cet article:

Costa, J. A. (2019). L'enseignement de la modalité linguistique en primaire - des voies méthodologiques possibles et souhaitables. Bellaterra Journal of Teaching \& Learning Language \& Literature 12(2), 41-59. DOI: https://doi.org/10.5565/rev/jt13.810
\end{abstract}

